\title{
Broadband active sound control system for air-conditioning duct noise
}

\author{
Minoru Takahashi,* Takashi Kuribayashi,* Kinichiro Asami,* \\ Takashi Enokida, ${ }^{* *}$ Hareo Hamada,** and Tanetoshi Miura** \\ * Research Laboratory, Hitachi Plant Engineering \& Construction Co., Ltd., \\ 537, Kami-Hongo, Matsudo, 271 Japan \\ **Tokyo Denki University, \\ 2-2, Kanda-Nishiki-cho, Chiyoda-ku, Tokyo, 101 Japan
}

(Received 4 August 1987)

\begin{abstract}
This paper describes an active sound control system using an FIR digital filter, which attenuate low-frequency noise in an air-conditioning duct system. This system is a monopole type with two identical microphones located on upstream and downstream sides at equal distances from the cancellation loudspeaker in the duct. The output signals of the two microphones are mixed $180^{\circ}$ out of phase with one another, and are fed to the input terminal of the digital filter. This reduces acoustic feedback in the system, offering high performance for duct noise caused by a centrifugal fan in a range of $70 \mathrm{~Hz}$ to $600 \mathrm{~Hz}$.
\end{abstract}

PACS number: 43. 50. Gf

\section{INTRODUCTION}

For better noise control of an air-conditioning duct system as in auditoriums, hospitals, and large buildings, new ways of achieving high attenuation of noise, particularly at low frequencies, are desired. ${ }^{1)}$

Many researchers have recently focused on active silencers using digital signal processing techniques as an alternative to traditional reactive silencers. ${ }^{2-6)}$

Okda et al. analyzed the principle of active control of noise using impedance. ${ }^{7)}$ In the paper, theoretical treatment of acoustical feedback for a multimicrophone and multiloudspeaker system was explained.

To avoid acoustic feedback, Kido et al. proposed a method of synthesized sound. ${ }^{8)}$ In their system, the loudspeaker is set close the end of a duct. Jessel and Mangiante ${ }^{9)}$ presented a tripole noise sources system for making an undirectional source, and Swinbanks ${ }^{10)}$ proposed an active silencer using a dipole noise sources system.

A new active silencer for practical use in a duct system that avoids acoustical feedback problem in a simple manner is desired.
This paper describes a simple active silencer operating on dual sensing microphone (DSM) principles to suppress acoustic feedback, and reports on the results of its applications to broadband random noise and air-conditioning duct noise. ${ }^{11)}$

The adaptive version of DSM active silencer ${ }^{12)}$ using fast least-mean-squares (FLMS) ${ }^{13)}$ algorithm will be described in the following paper.

\section{MODELING OF ORDINARY MONOPOLE ACTIVE SILENCER}

In addition to noise propagation in ducts, correction of the characteristics of electric transducers, such as microphones and loudspeakers, is important to ensure maximum performance of an active silencer.

Figure 1 shows the typical configuration of an ordinary monopole active silencer. In Fig. 1, $G_{d}$ denotes the acoustical transfer functions from the sound pressure at sensing microphone $M_{1}$ to the sound pressure at monitoring microphone $\mathrm{M}_{2}, G_{\mathrm{d}}{ }^{\prime}$ and $G_{\mathrm{t}}$ also denote the acoustical transfer functions from appropriate output points of loudspeaker $\mathbf{S}$ 


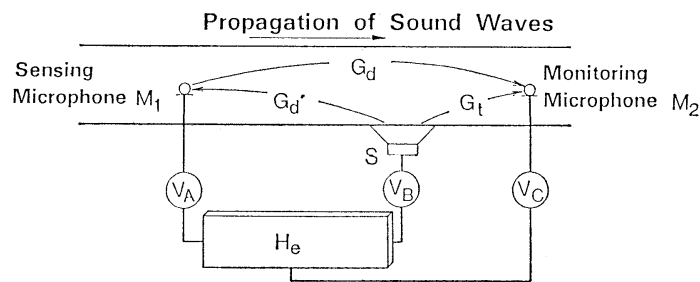

Fig. 1 Ordinary monopole active silencer configuration.

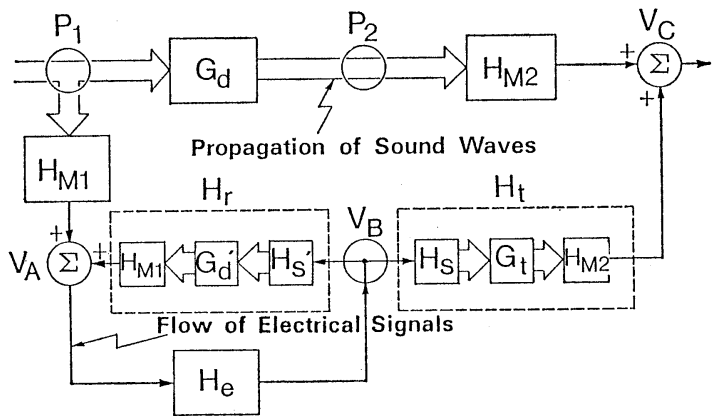

Fig. 2 Ordinary monopole active silencer block diagram. $P_{1}$ : Sound pressure at the $\mathrm{M}_{1}, H_{\mathrm{M} 1}$ : acoustic to electric transfer characteristic of $\mathrm{M}_{1}, P_{2}$ : Sound Pressure at the $\mathrm{M}_{2}, H_{\mathrm{M} 2}$ : Acoustic to electric transfer characteristic of $\mathrm{M}_{2}, V_{\mathrm{A}}, V_{\mathrm{B}}, V_{\mathrm{C}}$ : Voltage at respective points.

to microphone $\mathrm{M}_{1}$ and $\mathrm{M}_{2}$ respectively. $V_{\mathrm{A}}$ is the output voltage of sensing microphone $\mathrm{M}_{1}$. Also $V_{\mathrm{B}}$ and $V_{\mathrm{C}}$ are voltages of the respective points. $H_{\mathrm{e}}$ denotes the transfer function of the controller.

Assume that the sound field in the duct is one dimensional, and the effective diameter of the transducer diaphragm is small compared with the wave length.

From the above assumptions, the model approach using electric block diagram shown in Fig. 2 is made possible.

Transfer function $H_{\mathrm{r}}$ defined in Fig. 2 includes the electric-acoustic transfer characteristic of the loudspeaker $\mathrm{S}\left(H_{\mathrm{s}}{ }^{\prime}\right)$, sound propagation $\left(G_{\mathrm{d}}{ }^{\prime}\right)$ and the acoustic-electric transfer characteristic of the microphone $\mathrm{M}_{1}\left(H_{\mathrm{M} 1}{ }^{\prime}\right)$. Another transfer function $H_{\mathrm{t}}$ is defined similarly.

Sound pressure, $\boldsymbol{P}_{2}$, in the location of a monitor microphone, and voltages, $V_{\mathrm{A}}, V_{\mathrm{B}}$, and $V_{\mathrm{C}}$, at measuring points are expressed as follows:

$$
\begin{aligned}
& P_{2}=P_{1} \cdot G_{\mathrm{d}} \\
& V_{\mathrm{A}}=P_{1} \cdot H_{\mathrm{M} 1}+V_{\mathrm{B}} \cdot H_{\mathrm{r}} \\
& V_{\mathrm{B}}=V_{\mathrm{A}} \cdot H_{\mathrm{e}} \\
& V_{\mathrm{C}}=P_{2} \cdot H_{\mathrm{M} 2}+V_{\mathrm{B}} \cdot H_{\mathrm{t}}
\end{aligned}
$$

From Eqs. (2) and (3), $V_{A}$ can be expressed as follows:

$$
V_{\mathrm{A}}=\frac{P_{1} \cdot H_{\mathrm{M} 1}}{1-H_{\mathrm{e}} \cdot H_{\mathrm{r}}}
$$

Similarly, from Eqs. (1), (3) and (5), $V_{\mathrm{C}}$ can be expressed as follows:

$$
V_{\mathrm{C}}=P_{1}\left(G_{\mathrm{d}} \cdot H_{\mathrm{M} 2}+\frac{H_{\mathrm{M} 1} \cdot H_{\mathrm{e}} \cdot H_{\mathrm{t}}}{1-H_{\mathrm{e}} \cdot H_{\mathrm{r}}}\right)
$$

Here, for $V_{\mathrm{C}}=0$, the following equation is obtained from Eq. (6):

$$
G_{\mathrm{d}} \cdot H_{\mathrm{M} 2}=-\frac{H_{\mathrm{M} 1} \cdot H_{\mathrm{e}} \cdot H_{\mathrm{t}}}{1-H_{\mathrm{e}} \cdot H_{\mathrm{r}}}
$$

As a result, the transfer function, $H_{\mathrm{e}}$, can be expressed as follows:

$$
H_{\mathrm{e}}=\frac{-G_{\mathrm{d}} \cdot \frac{H_{\mathrm{M} 2}}{H_{\mathrm{M} 1}}}{H_{\mathrm{t}}-G_{\mathrm{d}} \cdot \frac{H_{\mathrm{M} 2}}{H_{\mathrm{M} 1}} \cdot H_{\mathrm{r}}}
$$

The transfer functions of $G_{\mathrm{d}} \cdot\left(H_{\mathrm{M} 2} / H_{\mathrm{M} 1}\right), H_{\mathrm{t}}$, and $H_{\mathrm{r}}$ are necessary to determine $H_{\mathrm{e}}$, and are all estimated using $V_{\mathrm{A}}, V_{\mathrm{B}}$, and $V_{\mathrm{C}}$ at measured points.

The suppression of acoustic feedback from loudspeaker $\mathrm{S}$ to microphone $M_{1}$ is essential to implement an ordinary monopole active silencer.

Possible methods for suppressing acoustic feedback are as follows:

(1) Installation of a low-frequency sound absorption layer between an additional sound source and a sensor microphone to reduce feedback sound pressure;

(2) Installation of an additional sound source and a sensor microphone sufficiently distant from each other to attenuate feedback sound pressure; and

(3) Use of a directive microphone as a sensor microphone to suppress the collection of additional sound, or, use of a directive sound source as an additional sound source.

Acoustic feedback suppression methods based on these principles have difficulties because the additional sound itself is a low-frequency sound. Several acoustic feedback suppression methods have been proposed, but they need to be improved to meet the trend of increases in the number of electroacousti- 


\section{TAKAHASHI et al.: ACTIVE SOUND CONTROL FOR DUCT NOISE}

cal transducers used and complexity of electronic circuits.

\section{DSM ACTIVE SILENCER}

We propose the DSM active silenser for improving the above mentioned problems. Figure 3 shows its system configuration. The difference from the ordinary monopole active silencer is only the addition of a differential amplifier. The monitoring microphone, $\mathbf{M}_{2}$, also serves as a sensor microphone.

In the DSM active silencer, transfer function $H_{\mathrm{r}}$ is made equal to transfer function $H_{\mathrm{t}}$ for electrical interference at an input terminal of the controller, consequently the acoustic feedback from the loudspeaker is suppressed. Various application experiments confirmed that this principle could be practically applied only by placing a loudspeaker at the center of two microphones. Chap. 5 reports on measurement results for suppression of acoustic feedback.

Figure 4 is a block diagram of the DSM active silencer. Transfer function $H_{\mathrm{e}}$, which determines the control characteristic of the controller, is derived below from Fig. 4.

Sound pressure $P_{2}$, at the location of the monitor microphone, and voltages $V_{\mathrm{A}}, V_{\mathrm{B}}$ and $V_{\mathrm{C}}$, at measur-

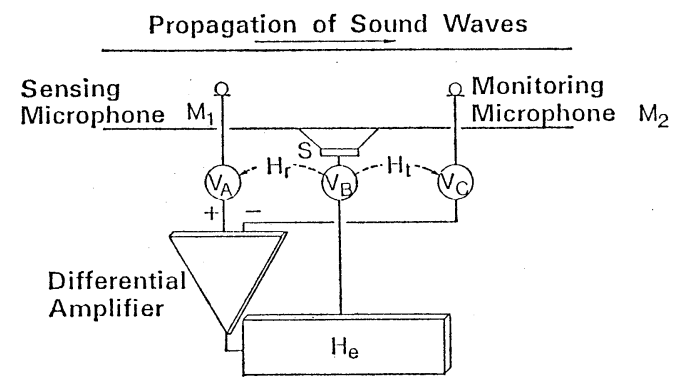

Fig. 3 DSM active silencer configuration.

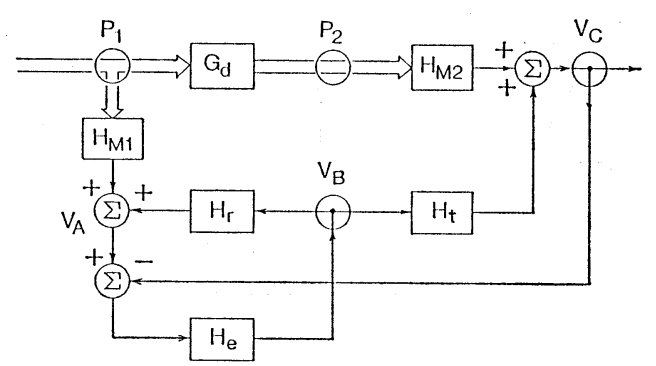

Fig. 4 DSM active silencer block diagram. ing points are expressed as follows:

$$
\begin{aligned}
& P_{2}=P_{1} \cdot G_{\mathrm{d}} \\
& V_{\mathrm{A}}=P_{1} \cdot H_{\mathrm{M} 1}+V_{\mathrm{B}} \cdot H_{\mathrm{r}} \\
& V_{\mathrm{B}}=\left(V_{\mathrm{A}}-V_{\mathrm{C}}\right) \cdot H_{\mathrm{e}} \\
& V_{\mathrm{C}}=P_{2} \cdot H_{\mathrm{M} 2}+V_{\mathrm{B}} \cdot H_{\mathrm{t}}
\end{aligned}
$$

From Eqs. (10) and (11), $V_{\mathrm{B}}$ can be expressed as follows:

$$
V_{\mathrm{B}}=\frac{P_{2} \cdot H_{\mathrm{M} 1} \cdot H_{\mathrm{e}}-V_{\mathrm{C}} \cdot H_{\mathrm{e}}}{1-H_{\mathrm{e}} \cdot H_{\mathrm{r}}}
$$

Similarly, from Eqs. (12) and (13), $V_{\mathrm{C}}$ can be expressed as follows:

$$
V_{\mathrm{C}}=\frac{P_{2} \cdot H_{\mathrm{M} 2}\left(1-H_{\mathrm{e}} \cdot H_{\mathrm{r}}\right)+P_{1} \cdot H_{\mathrm{M} 1} \cdot H_{\mathrm{e}} \cdot H_{\mathrm{t}}}{1-H_{\mathrm{e}}\left(H_{\mathrm{r}}-H_{\mathrm{t}}\right)}
$$

Also, by substituting Eq. (9), Eq. (14) can be expressed as follows:

$$
V_{\mathrm{C}}=\frac{P_{1}\left[H_{\mathrm{M} 1} \cdot H_{\mathrm{e}} \cdot H_{\mathrm{t}}+G_{\mathrm{d}} \cdot H_{\mathrm{M} 2}\left(1-H_{\mathrm{e}} \cdot H_{\mathrm{r}}\right)\right]}{1-H_{\mathrm{e}}\left(H_{\mathrm{r}}-H_{\mathrm{t}}\right)}
$$

Here, for $V_{\mathrm{C}}=0$, the following equation is obtained from Eq. (15).

$$
H_{\mathrm{e}}\left(H_{\mathrm{M} 1} \cdot H_{\mathrm{t}}-G_{\mathrm{d}} \cdot H_{\mathrm{M} 2} \cdot H_{\mathrm{r}}\right)=-G_{\mathrm{d}} \cdot H_{\mathrm{M} 2}
$$

As a result of this, the transfer function, $H_{\mathrm{e}}$, can be expressed as follows:

$$
H_{\mathrm{e}}=\frac{-G_{\mathrm{d}} \cdot \frac{H_{\mathrm{M} 2}}{H_{\mathrm{M} 1}}}{H_{\mathrm{t}}-G_{\mathrm{d}} \cdot \frac{H_{\mathrm{M} 2}}{H_{\mathrm{M} 1}} \cdot H_{\mathrm{r}}}
$$

As can be seen from Eq. (17), transfer functions $G_{\mathrm{d}} \cdot\left(H_{\mathrm{M} 2} / H_{\mathrm{M} 1}\right), H_{\mathrm{t}}$ and $H_{\mathrm{r}}$ are necessary to determine transfer function $H_{\mathrm{e}}$. As mentioned before, these transfer functions can be easily estimated, using $V_{\mathrm{A}}$, $V_{\mathrm{B}}$, and $V_{\mathrm{C}}$.

\section{DESIGN OF DIGITAL FILTER FOR CONTROLLER}

Suppose that all DSM active silencer components including a loudspeaker, two microphones, and acoustic properties in air-conditioning ducts are linear time-invariant.

We wish to model the controller as a finite impulse response (FIR) linear filter. If we assume that time domain representation of the denominator of Eq. (17) and that of the numerator express the observed output and the input of the controller respectively, and both of them are finite length sequences, the system identification problem then, is to determine the coefficients of moving average linear operator 
(an FIR filter) whose output is a least squared error estimate of the observed output of the controller, given the same input signal. The least square algorithm (LSA), ${ }^{14)}$ a well known filter design algorithm in the time domain, has been applied. Approximated filter coefficients $\boldsymbol{H}$ in vector form can be obtained by LSA as follows:

$$
\boldsymbol{H}=\left(X^{\mathrm{t}} X\right)^{-1} \cdot X^{\mathrm{t}} \boldsymbol{Y}
$$

Where $X$ is the matrix of which rows are composed by the shifted finite length input of the system. $Y$ is the finite length output sequence, and $t$ denotes transpose.

$X^{\mathrm{t}} X$ is an auto-correlation matrix of the input time sequence, and is symmetric and toeplitz, while $X^{\mathrm{t}} \boldsymbol{Y}$ is a cross-correlation vector of the input time sequence to the output time sequence. Therefore, we can use the FFT and Levinson algorithm ${ }^{15)}$ to calculate $\boldsymbol{H}$ for efficient computations and reduced memory size.

\section{DEMONSTRATION AGAINST VARIOUS NOISE SOURCES}

\subsection{Experimental Apparatus}

Figure 5 shows the experimental apparatus.

Experimental apparatus was built in a typical office, i.e., width $13 \mathrm{~m}$, length $15 \mathrm{~m}$ and height 2.8 m. Table 1 lists the absorption coefficients. An airconditioning duct, used in the sound cancellation experiment was $350 \mathrm{~mm}$ square $\times 13 \mathrm{~m}$ long. An additional sound source was installed at about the longitudinal center of the duct. The microphone $\mathrm{M}_{1}$ and $\mathrm{M}_{2}$ were $3 \mathrm{~m}$ apart from each other.

Table 1 Absorption coefficients of the office.

\begin{tabular}{|c|c|c|c|c|c|c|}
\hline & \multicolumn{5}{|c|}{ Octave band center frequency } & \multirow{2}{*}{$\frac{[\mathrm{Hz}]}{2,000}$} \\
\hline & 63 & 125 & 250 & 500 & 1,000 & \\
\hline $\begin{array}{l}\text { Absorption } \\
\text { coefficients }\end{array}$ & 0.19 & 0.20 & 0.21 & 0.16 & 0.15 & 0.15 \\
\hline
\end{tabular}

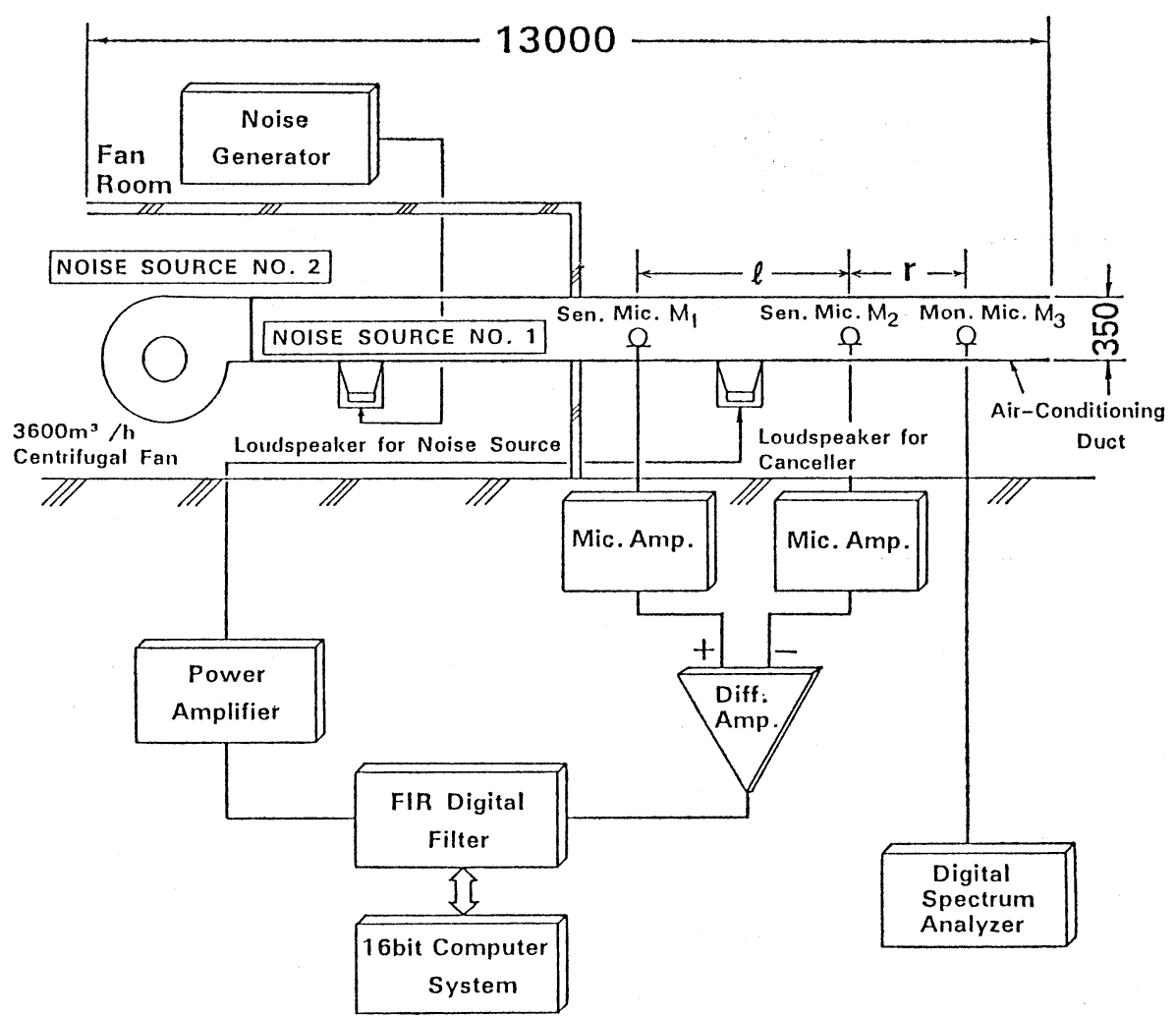

Fig. 5 Experimental apparatus for attenuations of sound cancellation. 
Noise sources No. 1 and No. 2 were used for the experiment. Noise source No. 1 is a loudspeaker driven by the random noise of which the frequency band is between $20 \mathrm{~Hz}$ and $1 \mathrm{kHz}$, and noise source No. 2 is a centrifugal fan of $3,600 \mathrm{~m}^{3} / \mathrm{h}$ in air-flow volume and $2,450 \mathrm{~Pa}$ in static pressure.

A realtime FIR digital filter (Digital Audio Corp. type PDF-1024) was used to produce an additional sound. The digital filter is $3.2 \mathrm{kHz}$ in sampling frequency and 512 points in coefficient length.

\subsection{Measured Result of Suppression of Acoustic Feedback}

Figure 6 shows an example of the closed loop gain of the DSM active silencer which is measured using the cross spectrum method.

$H_{\mathrm{e}} \cdot\left(H_{\mathrm{r}}-H_{\mathrm{t}}\right)$ denotes the closed loop gain of the DSM active silencer; $H_{\mathrm{e}} H_{\mathrm{r}}$ denotes the closed loop gain of the ordinary monopole active silencer. The amplitude of closed loop gain in the DSM active silencer can be reduced to less than $1(0 \mathrm{~dB})$ in all desired frequency bands. Therefore the system is substantially stable.

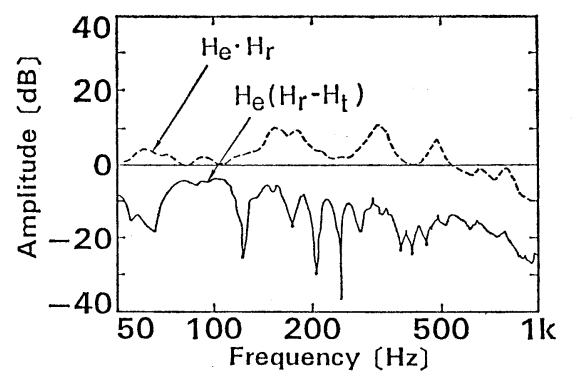

Fig. 6 Suppression of acoustic feedback using DSM active silencer.

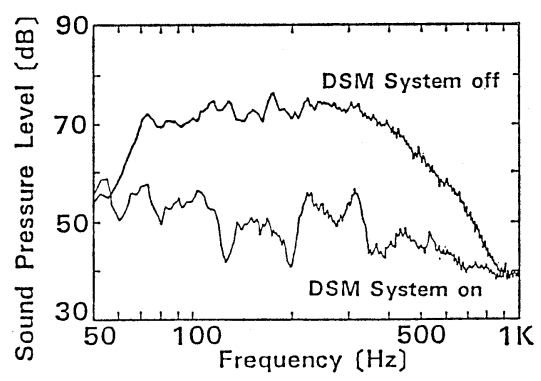

Fig. 7 Effects of sound cancellation for broadband random noise, measuring point $r=1.5 \mathrm{~m}$.
5.3 Demonstration against Broadband Random Noise

Figure 7 shows the noise spectrum at the microphone $\mathrm{M}_{2}$, before and after active control using the DSM system. The wide bandwidth and high level (over $15 \mathrm{~dB}$ ) of cancellation is observed in Fig. 7.

Figure 8 shows the distribution of the attenuation level. Seen from the figure, almost same attenuation level is obtained at the downward points.

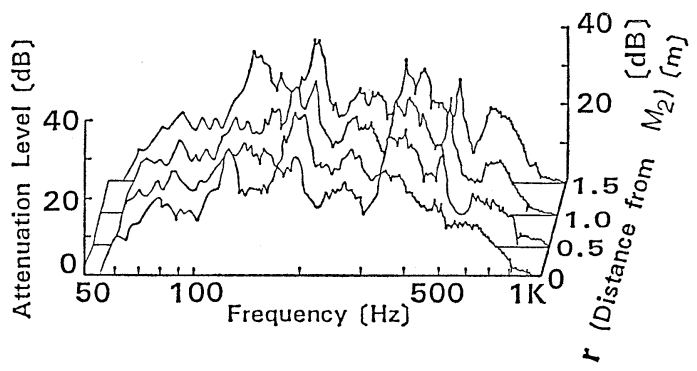

Fig. 8 Distribution of attenuation level.

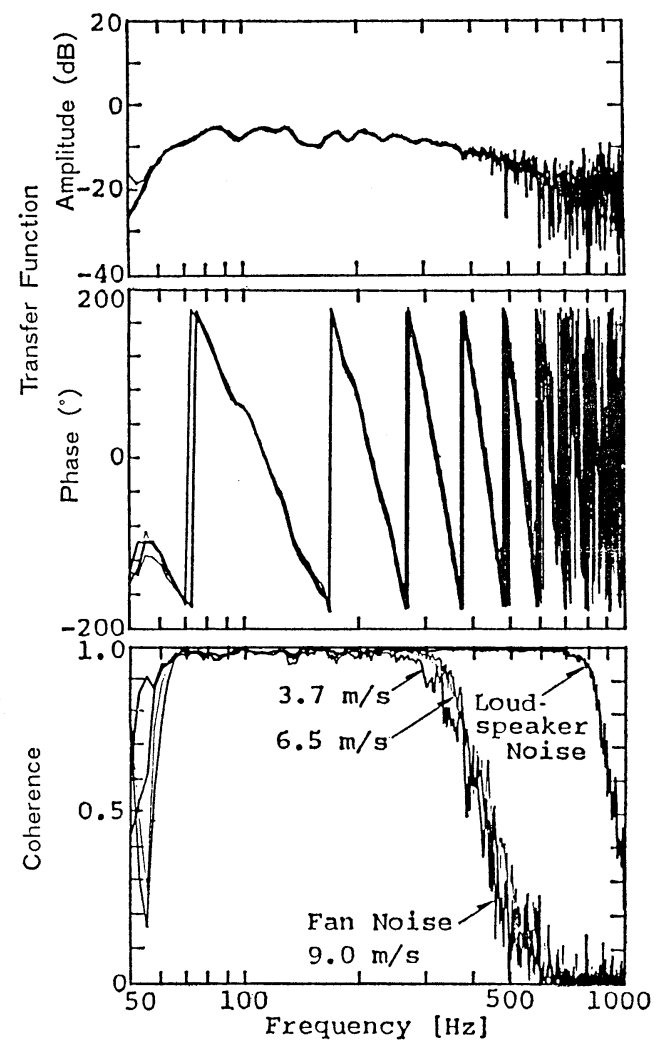

Fig. 9 Measured values of transfer function and coherence in straight ducts using two type noise sources. 


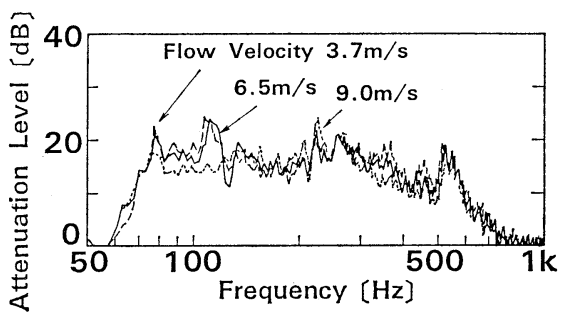

Fig. 10 Effects of sound cancellation for centrifugal fan noise, measuring point $r=$ $1.5 \mathrm{~m}$.

\subsection{Demonstration against Fan Noise}

Fan noise attenuations were measured using a centrifugal fan $\left(Q: 3,600 \mathrm{~m}^{3} / \mathrm{h} ; P: 2,450 \mathrm{~Pa}\right)$ as a noise source, and varying the induct flow velocity between 2.6 and $9.5 \mathrm{~m} / \mathrm{s}$. Figure 9 shows the measured transfer functions $G_{\mathrm{d}} \cdot\left(H_{\mathrm{M} 2} / H_{\mathrm{M} 1}\right)$ with noise source No. 1 (loudspeaker) and No. 2 (centrifugal fan), by the cross spectrum mothod. Within the limits of this experiment, the transfer function measured with noise source No. 1 agree well with that measured with noise source No. 2 in the range of $50 \mathrm{~Hz}$ to $300 \mathrm{~Hz}$, majority spectrum of the noise.

From this results, the same transfer function of the controller (FIR filter) as used in the previous cancellation experiment was used disregarding flow velocity.

Figure 10 shows the attenuation of the fan noise measured at the microphone $\mathrm{M}_{2}$.

The figure shows that more than $15 \mathrm{~dB}$ of attenuation can be obtained even in the presence of air flow, and the effect of attenuation is slightly reduced with rapid increases in the flow velocity.

We are also now carrying out research into adaptive control of a DSM active silencer capable of following up changes in various transfer functions associated with flow velocity, internal pressure, temperature, and so on.

\section{CONCLUSIONS}

An active silencer modeling method was described, assuming that ducts, electroacoustical transducer, and other components of the active silencer for airconditioning duct are linear time-invariant. Based on this method, we proposed a new monopole system called "DSM active silencer."

Further, we designed a digital filter for a controller in the DSM active silencer, using a realtime FIR digital filter.

Next, we installed this system in an actual airconditioning duct $(350 \mathrm{~mm}$ square $\times 13 \mathrm{~m}$ long) and made sound cancellation experiments. To summarize the results of our experiments, we can conclude that more than $15 \mathrm{~dB}$ of attenuation can be obtained and which extends from $70 \mathrm{~Hz}$ to about $600 \mathrm{~Hz}$ for broadband random noise, even in the presence of air flows $(3.7$ to $9.0 \mathrm{~m} / \mathrm{s})$ in the actual duct.

We will carry out research into improving the reliability of hardware and promoting ongoing research of the active sound control system to reach a stage where the system, including the adaptive DSM active silencer, becomes practically useful.

\section{ACKNOWLEDGMENTS}

We wish to thank Mr. Yoshitaka Oguri, General Manager of the Research Laboratory of Hitachi Plant Engineering \& Construction Co., Ltd. for his helpful advice and suggestions.

\section{REFERENCES}

1) M. Takahashi and K. Asami, "Noise level prediction method for air-conditioning duct system," Proc. Inter-Noise 81, 497-502 (1981).

2) G. B. B. Chaplin, "The cancellation of repetitive noise and vibration," Proc. Inter-Noise 80, 699702 (1980).

3) C. F. Ross, "An adaptive digital filter for broadband active sound control," J. Sound Vib. 80, 381388 (1982).

4) G. E. Warnaka, J. Tichy, J. M. Zalas, and L. A. Pool, "Active control of noise in interior spaces," Proc. Inter-Noise 80, 415-418 (1980).

5) T. Kosaka and S. Yamada, "Active attenuation of repetitive low frequency noise in pipe," J. Acoust. Soc. Jpn. (J) 41, 316-321 (1985).

6) A. Roure, "Self-adaptive broadband active sound control system," J. Sound Vib. 101, 411-429 (1985).

7) J. Okda, T. Usagawa, and M. Ebata, "Analysis of active control of noise in duct using impedance," J. Acoust. Soc. Jpn. (E) 8, 63-72 (1987).

8) K. Kido, S. Morikawa, and M. Abe, "Stable method for active cancellation of duct noise by synthesized sound," Trans. ASME 109, 37-42 (1987).

9) M. J. M. Jessel and G. A. Mangiante, "Active sound absorbers in an air duct," J. Sound Vib. 23, 383390 (1972).

10) M. A. Swinbanks, "The active control of sound propagation in long ducts," J. Sound Vib. 27, 411436 (1973).

11) M. Takahashi, T. Kuribayashi, K. Asami, H. Hamada, T. Enokida, and T. Miura, "Electric sound 


\section{TAKAHASHI et al:: ACTIVE SOUND CONTROL FOR DUCT NOISE}

cancellation in air-conditioning duct system," Proc. Inter-Noise 86, 607-610 (1986).

12) T. Enokida, H. Hamada, T. Miura, M. Takahashi, T. Kuribayashi, and K. Asami, "Design of electric sound cancellation system for air-conditioning ducts," Proc. Inter-Noise 86, 589-594 (1986).

13) C. F. N. Cowan and P. M. Grant, Adaptive Filters
(Prentice-Hall, 1985), p. 180.

14) B. A. Bowen and W. R. Brown, VLSI System Design for Digital Signal Processing, Vol. 1 (PrenticeHall, 1982), Chap. 3.

15) R. E. Blahut, Fast Algorithms for Digital Signal Processing (Addison-Wesley, 1985), p. 352. 